\title{
Higgs Mass Bounds in the Three- and Six-Higgs Doublet Models for Family Structure
}

Stephen L. Adler

Institute for Advanced Study

Princeton, NJ 08540

Send correspondence to:

Stephen L. Adler

Institute for Advanced Study

Olden Lane, Princeton, NJ 08540

Phone 609-734-8051; FAX 609-924-8399; email adler@ias.edu 


\begin{abstract}
We reanalyze our recently proposed mass matrix model based on spontaneously broken discrete chiral family symmetry, taking into account the additional flavor changing neutral current constraint implied by the bound on the $D_{1}-D_{2}$ mass difference, and including several corrections to our earlier analysis. When combined, the $K_{1}-K_{2}$ and $D_{1}-D_{2}$ constraints force the masses of the Higgs particles that contribute most strongly to flavor changing neutral currents (the $\phi$ Higgs states) to lie above $17 \mathrm{TeV}$, well beyond the limit of validity of conventional perturbative Higgs physics. The analogous constraints on the masses of the $\eta$ Higgs states and the neutral pseudo Goldstone Higgs state depend on the mechanism for realizing small first family masses. If the $\eta$ Higgs is the primary contributor to second family masses, the pseudo Goldstone and $\eta$ Higgs states must have masses above $220 \mathrm{GeV}$, with numerical fits suggesting masses above $1 \mathrm{TeV}$, while if the $\eta$ Higgs is responsible solely for first family masses, the corresponding mass bounds drop to the range detectable at the LHC. We show that naturalness of small first family masses favors the latter alternative, and give an illustrative mass matrix texture model.
\end{abstract}




\section{Introduction}

In a recent paper [1] we constructed extensions of the standard model, based on the hypothesis that the Higgs bosons also exhibit a threefold family structure, and that the flavor weak eigenstates are distinguished by a discrete $Z_{6}$ chiral symmetry that is spontaneously broken by the Higgs sector. Two models were analyzed in [1], the first with one three-family set of Higgs doublets, and the second with two three-family sets of Higgs doublets. In the three-Higgs doublet model, the leading cyclically symmetric approximation to the quark and lepton mass matrices has the "democratic" form with all matrix elements equal, leading to one massive and two massless fermion families. In the six-Higgs doublet model, for a wide range of Higgs potential parameters, $C P$ is spontaneously broken, and this breaking simultaneously modifies the democratic Ansatz to give nonzero masses to an additional family (assumed in [1] to be the second family) in leading cyclic approximation. Corrections to the cyclic approximation were used in [1] to give first family masses, and a nontrivial CKM matrix.

In performing numerical fits to the data using the models of [1], we took into account bounds on flavor changing neutral currents solely through the constraint provided by the $K_{1}-K_{2}$ mass difference, which led to strong asymmetries in the fits between the up and down quark sectors. M. Peskin [2] has pointed out the importance of including in the analysis experimental bounds on the $D_{1}-D_{2}$ mass difference, which is the up quark sector analog of the $K_{1}-K_{2}$ mass difference constraint. The purpose of this paper is to give the formulas and numerical results needed for this extension of the analysis of [1]. We also consider an alternative version of the model of [1], in which cyclic asymmetries in the $\phi$ Higgs couplings are responsible for second family masses, and the $\eta$ Higgs contributes significantly only to 
first family masses. We show that this alternative is favored by requiring naturalness of small first family masses. In addition, we make the following three corrections to the model as originally formulated: (i) we correct the form of the CKM matrix, as pointed out in an Erratum [3] to [1], (ii) we include rephasings needed to make the diagonalized quark mass matrices positive real, and (iii) we correct combinatoric factors in the flavor changing neutral current amplitude (amounting to an overall factor of 2), and give a more accurate treatment of the hadronic matrix elements appearing in the flavor changing neutral current constraints.

This paper is organized as follows. In Sec. II we give a synopsis of results needed from [1], including the corrections (i) and (ii) noted above. In Secs. III and IV we analyze $K_{1}-K_{2}$ and $D_{1}-D_{2}$ mixing induced by Higgs exchange, including the corrections (iii). In Sec. III we give formulas for calculating the $\phi$ Higgs, the $\eta$ Higgs, and the pseudo Goldstone Higgs contributions, in the six-Higgs doublet model, to both the $K_{1}-K_{2}$ and $D_{1}-D_{2}$ mass differences. In Sec. IV we use the formulas of Secs. II and III to derive a series of bounds on the Higgs masses, which are evaluated numerically using lattice and model calculations of the relevant hadronic matrix elements, for two possible mechanisms for realizing the first and second family masses. Irrespective of this choice of mechanism, we find that the $\phi$ Higgs masses must be greater than $17 \mathrm{TeV}$, in accord with analyses [4] of generic multi-Higgs models. This bound also extends, by specialization to the case in which the $\eta$ Higgs couplings vanish, to the CP conserving case of the three-Higgs doublet model. In Sec. V we analyze the implications of requiring that small first family masses arise naturally, as opposed to arising by detailed cancellations between physically unrelated quantities, and give a simple mass matrix texture model corresponding to the case in which Yukawa coupling asymmetries are responsible for second family masses. In Sec. VI we repeat the numerical 
fits of [1], taking into account the results derived in the preceding sections, and summarize our conclusions.

\section{Synopsis of Needed Results from the Six-Higgs Model}

The six-Higgs doublet model of [1] is based on the assumption that there are two discrete chiral families of Higgs bosons, $\phi_{n}$ and $\eta_{n}, n=1,2,3$. These are coupled to discrete chiral families of fermions to give a Lagrangian that is exactly discrete chiral invariant, and that is approximately invariant under cyclic permutation of the discrete chiral components. The model is constructed so that the Higgs fields develop nonvanishing vacuum expectations in a CP violating phase, and it is assumed that the $\phi$ Higgs bosons couple much more strongly to fermions than the $\eta$ Higgs bosons, and similarly for their corresponding expectations (denoted respectively by $\Omega_{\phi}$ and $\Omega_{\eta}$ ). As a zeroth order approximation to the model, only the $\phi$ Higgs expectations are retained and cyclic permutation symmetry is assumed, leading to a "democratic" mass matrix with one massive and two massless families, and a CKM matrix of unity.

Deviations from cyclic symmetry, and the $\eta$ Higgs expectations, are then added back as a perturbation, giving as the Lagrangian mass term

$$
\mathcal{L}_{\text {mass }}=\sum_{f=u, d, e} \bar{f}_{L}^{\prime} g_{\phi}^{f} \Omega_{\phi}\left(3 M^{(3)}+\sigma^{f}\right) f_{R}^{\prime}
$$

Here $M^{(3)}=\operatorname{diag}(0,0,1)$ is the projector on the third family, $g_{\phi}^{f}$ is the $\phi$ Higgs Yukawa 
coupling for flavor $f$, and $\sigma^{f}$ is a $3 \times 3$ matrix of perturbations, given explicitly by

$$
\begin{aligned}
\sigma_{11}^{f} & =\frac{1}{3} \mu_{11}^{f}+\delta_{3}^{f}+\bar{\omega} \delta_{2}^{f}+\omega \delta_{1}^{f}, \\
\sigma_{22}^{f} & =\frac{1}{3} \mu_{22}^{f}+3 R^{f}+\delta_{3}^{f}+\omega \delta_{2}^{f}+\bar{\omega} \delta_{1}^{f}, \\
\sigma_{33}^{f} & =0, \\
\sigma_{\ell m}^{f} & =\frac{1}{3} \mu_{\ell m}^{f}, \quad \ell \neq m ; \quad \omega \equiv \exp (2 \pi i / 3), \quad \bar{\omega} \equiv \omega^{*} .
\end{aligned}
$$

In Eq. (1b) the terms $\mu_{\ell m}$ arise from small deviations from cyclic symmetry in the Yukawa couplings of the $\phi$ Higgs bosons, the terms $\delta_{n}^{f}$ arise from deviations from cyclic symmetry in the $\phi$ Higgs expectations, and the term $R^{f}$ arises from contributions to the mass matrix of the weakly coupled $\eta$ Higgs expectations. Further details of the structure of $\sigma^{f}$ are given in Eqs. (38b) through (39c) of [1] and are used in Secs. V and VI below. However, the only property needed for the analytic calculations of Sec. III is that, since the Yukawa coupling asymmetries are all real because the Lagrangian in the six-Higgs doublet model is assumed to be CP invariant, the first order perturbations $\sigma^{f}$ obey

$$
\sigma_{12}^{f}=\sigma_{21}^{f *}
$$

This restriction holds even though the model of [1] chooses a CP violating ground state.

Defining

$$
M_{f}^{\prime}=3 M^{(3)}+\sigma^{f}
$$

we then [1] construct the bi-unitary transformation matrices $U_{L}^{f}, \quad U_{R}^{f}$ for which $U_{L}^{f} M_{f}^{\prime} U_{R}^{f \dagger}$ is diagonal, with the eigenvalues ordered in absolute value. The fermion mass eigenstate basis, up to rephasings to be discussed, is related to the primed basis by

$$
\begin{aligned}
& f_{L}^{\prime}=U_{L}^{f \dagger} f_{L}^{\text {mass }}, \\
& f_{R}^{\prime}=U_{R}^{f \dagger} f_{R}^{\text {mass }} \quad, \quad f=u, d, e,
\end{aligned}
$$


and the CKM matrix $U_{\mathrm{CKM}}$ is given [3] by

$$
U_{\mathrm{CKM}}=U_{L}^{u} U_{L}^{d \dagger}
$$

Since Eq. (2a) defines a degenerate perturbation problem, the matrices $U_{L, R}^{f}$ are constructed [1] in two stages: first the $2 \times 2$ submatrix of $M_{f}^{\prime}$ spanned by the first two families is diagonalized exactly, and then the solution to this problem is used to perturbatively construct the full $3 \times 3$ diagonalizing matrices. Because the analysis of flavor changing neutral current effects in the next section ignores third family mixings, it suffices for this analysis to discuss only the $2 \times 2$ submatrix diagonalization problem. Suppressing for the time being the flavor superscript $f$, we define the $2 \times 2$ submatrix $m$ by

$$
m=\left(\begin{array}{ll}
\sigma_{11} & \sigma_{12} \\
\sigma_{21} & \sigma_{22}
\end{array}\right)
$$

which is brought to diagonal (but not necessarily real) form by matrices $V_{L, R}$,

$$
V_{L} m V_{R}^{\dagger}=\left(\begin{array}{cc}
\kappa_{1} & 0 \\
0 & \kappa_{2}
\end{array}\right)
$$

with $\left|\kappa_{1}\right| \leq\left|\kappa_{2}\right|$. An explicit construction of $V_{L, R}$ is given in Appendix B of [1]; the results obtained there can be simplified by using the symmetry of Eq. (1c) above, which (in terms of the quantities defined in Appendix B of [1]) implies that

$$
\begin{aligned}
& A_{L}=A_{R}=\left|\sigma_{11}\right|^{2}+\left|\sigma_{12}\right|^{2} \equiv A \\
& B_{L}=B_{R}=\left|\sigma_{22}\right|^{2}+\left|\sigma_{12}\right|^{2} \equiv B, \\
& z_{L}=\left(\sigma_{11}^{*}+\sigma_{22}\right) \sigma_{12}^{*}, \quad z_{R}=\left(\sigma_{11}+\sigma_{22}^{*}\right) \sigma_{12}^{*}, \\
& \left|z_{L}\right|=\left|z_{R}\right| \equiv|z| .
\end{aligned}
$$

These relations, together with the results in Appendix B of [1], imply that

$$
V_{L, R}=\left(\begin{array}{cc}
\cos \Theta & -\exp \left(-i \phi_{L, R}\right) \sin \Theta \\
\exp \left(i \phi_{L, R}\right) \sin \Theta & \cos \Theta
\end{array}\right)
$$


with

$$
\exp \left(i \phi_{L, R}\right)=z_{L, R} /|z|
$$

and

$$
\Theta=\frac{1}{2} \tan ^{-1}\left(\frac{-2|z|}{A-B}\right)
$$

Although the construction just given suffices for the computation of the magnitudes of the CKM matrix elements, the calculation of the Higgs exchange amplitude in the next section requires care in the choices of phases. When we rephase the physical mass eigenstates $f_{L, R}^{\text {mass }}$, the matrices $V_{L, R}^{f}$ transform according to

$$
V_{L, R}^{f} \rightarrow \hat{V}_{L, R}^{f}=D_{L, R}^{f} V_{L, R}^{f}
$$

with $D_{L, R}^{f}$ diagonal unitary matrices. A correct choice of phases requires that the diagonalized mass matrix

$$
\hat{V}_{L}^{f} m^{f} \hat{V}_{R}^{f \dagger}
$$

be real and positive; in other words, the matrices $D_{L, R}^{f}$ must be chosen to absorb the phases of the diagonal matrix elements $\kappa_{1,2}^{f}$ on the right of Eq. (3b) above. In addition, restricting ourselves now to the up and down quark flavor sectors, we shall require that the phase choices for the physical states put the matrix $\hat{V}_{L}^{u} \hat{V}_{L}^{d \dagger}$, which is the $2 \times 2$ submatrix of the rephased CKM matrix when third family mixings are neglected, into the standard real form

$$
\left(\begin{array}{cc}
c_{12} & s_{12} \\
-s_{12} & c_{12}
\end{array}\right)
$$

with $s_{12}$ and $c_{12}$ both nonnegative. These two phase requirements together fix the rephasing matrices $D_{L, R}^{f}$ up to an irrelevant overall phase. 
To carry this construction out explicitly, we write Eq. (3b), in the up and down quark sectors, as

$$
V_{L}^{f} m^{f} V_{R}^{f \dagger}=\left(\begin{array}{cc}
\left|\kappa_{1}^{f}\right| \exp \left(i \theta_{1}^{f}\right) & 0 \\
0 & \left|\kappa_{2}^{f}\right| \exp \left(i \theta_{2}^{f}\right)
\end{array}\right), \quad f=u, d
$$

and we write the adjoint of the $2 \times 2$ CKM matrix computed before rephasing as

$$
V_{L}^{d} V_{L}^{u \dagger}=\left(\begin{array}{cc}
c_{12} \exp \left(i \theta_{11}\right) & -s_{12} \exp \left(i \theta_{12}\right) \\
s_{12} \exp \left(i \theta_{21}\right) & c_{12} \exp \left(i \theta_{22}\right)
\end{array}\right)
$$

with unitarity imposing the conditions

$$
\begin{aligned}
& c_{12}^{2}+s_{12}^{2}=1, \\
& \theta_{11}+\theta_{22}=\theta_{12}+\theta_{21}(\bmod 2 \pi) .
\end{aligned}
$$

Then a simple calculation gives

$$
\begin{aligned}
& D_{L}^{d}=\operatorname{diag}\left[\exp \left(-i \theta_{11}\right), \exp \left(-i \theta_{21}\right)\right] \\
& D_{R}^{d}=\operatorname{diag}\left[\exp \left(i \theta_{1}^{d}-i \theta_{11}\right), \exp \left(i \theta_{2}^{d}-i \theta_{21}\right)\right] \\
& D_{L}^{u}=\operatorname{diag}\left[1, \exp \left(-i \theta_{21}+i \theta_{22}\right)\right], \\
& D_{R}^{u}=\operatorname{diag}\left[\exp \left(i \theta_{1}^{u}\right), \exp \left(i \theta_{2}^{u}-i \theta_{21}+i \theta_{22}\right)\right]
\end{aligned}
$$

Corresponding to these, we find from Eqs. (4b) and (5a) that

$$
\begin{gathered}
\hat{V}_{L}^{d}=\left(\begin{array}{cc}
\exp \left(-i \theta_{11}\right) c_{d} & -\exp \left(-i \phi_{L}^{d}-i \theta_{11}\right) s_{d} \\
\exp \left(i \phi_{L}^{d}-i \theta_{21}\right) s_{d} & \exp \left(-i \theta_{21}\right) c_{d}
\end{array}\right) \\
\hat{V}_{R}^{d}=\left(\begin{array}{cc}
\exp \left(-i \theta_{11}+i \theta_{1}^{d}\right) c_{d} & -\exp \left(-i \phi_{R}^{d}-i \theta_{11}+i \theta_{1}^{d}\right) s_{d} \\
\exp \left(i \phi_{R}^{d}-i \theta_{21}+i \theta_{2}^{d}\right) s_{d} & \exp \left(-i \theta_{21}+i \theta_{2}^{d}\right) c_{d}
\end{array}\right) \\
\hat{V}_{L}^{u}=\left(\begin{array}{cc}
c_{u} & -\exp \left(-i \phi_{L}^{u}\right) s_{u} \\
\exp \left(i \phi_{L}^{u}-i \theta_{21}+i \theta_{22}\right) s_{u} & \exp \left(-i \theta_{21}+i \theta_{22}\right) c_{u}
\end{array}\right) \\
\hat{V}_{R}^{u}=\left(\begin{array}{cc}
\exp \left(i \theta_{1}^{u}\right) c_{u} & -\exp \left(-i \phi_{R}^{u}+i \theta_{1}^{u}\right) s_{u} \\
\exp \left(i \phi_{R}^{u}-i \theta_{21}+i \theta_{22}+i \theta_{2}^{u}\right) s_{u} & \exp \left(-i \theta_{21}+i \theta_{22}+i \theta_{2}^{u}\right) c_{u}
\end{array}\right)
\end{gathered}
$$

with $c_{d, u}, s_{d, u}$ defined in terms of the angle $\Theta$ of Eq. (4d) by

$$
c_{d, u}=\cos \left(\Theta_{d, u}\right), \quad s_{d, u}=\sin \left(\Theta_{d, u}\right)
$$


Equations (4a-d), (6a, b), and (7a-e) provide our starting point for calculating the Higgs exchange contributions to flavor changing neutral current processes.

\section{Analysis of $K_{1}-K_{2}$ and $D_{1}-D_{2}$ Mixing \\ Induced by Higgs Exchange}

We begin by extending the formulas of [1] for the Higgs exchange contribution to the $K_{1}-K_{2}$ mass difference to the case when the $\eta$ Higgs and pseudo Goldstone Higgs

contributions are also included, using the rephased matrices $\hat{V}_{L, R}^{u, d}$ of Eqs. (7a-e). Our starting point is Eq. (45c) of [1] for the $\Delta S=1$ terms in the Higgs Lagrangian density, calculated to zeroth order in the perturbation $\sigma^{f}$, which when extended to include the $\eta$ and pseudo Goldstone Higgs couplings reads

$$
\begin{aligned}
\mathcal{L}_{\mathrm{scnc}}^{\Delta S=1} & =\sum_{\xi=\phi, \eta} \sum_{p= \pm} \sum_{F=R, I}\left[\bar{d}_{\xi F}^{(p)}\left(A_{\xi F 12}^{(p)}+B_{\xi F 12}^{(p)} \gamma_{5}\right) s+\bar{s} \epsilon_{\xi F}^{(p)}\left(A_{\xi F 21}^{(p)}+B_{\xi F 21}^{(p)} \gamma_{5}\right) d\right] \\
& +\bar{d} \epsilon_{\eta R}^{(3)}\left(A_{\eta R 12}^{(3)}+B_{\eta R 12}^{(3)} \gamma_{5}\right) s+\bar{s} \epsilon_{\eta R}^{(3)}\left(A_{\eta R 21}^{(3)}+B_{\eta R 21}^{(3)} \gamma_{5}\right) d \\
& +\bar{d} \epsilon_{P G}^{(3)}\left(A_{P G 12}+B_{P G 12} \gamma_{5}\right) s+\bar{s} \epsilon_{P G}^{(3)}\left(A_{P G 21}+B_{P G 21} \gamma_{5}\right) d
\end{aligned}
$$

The corresponding formula for the effective Hamiltonian density $\mathcal{H}_{\text {eff }}^{\Delta S=2}$ for the $\Delta S=2$ process $s+s \rightarrow d+d$ is

$$
\begin{aligned}
\mathcal{H}_{\text {eff }}^{\Delta S=2} & =-\frac{1}{2} \sum_{\xi=\phi, \eta} \sum_{p= \pm} \sum_{F=R, I} \bar{d}\left(A_{\xi F 12}^{(p)}+B_{\xi F 12}^{(p)} \gamma_{5}\right) s \frac{1}{M_{\xi F}^{2(p)}} \bar{d}\left(A_{\xi F 12}^{(p)}+B_{\xi F 12}^{(p)} \gamma_{5}\right) s \\
& -\frac{1}{2} \bar{d}\left(A_{\eta R 12}^{(3)}+B_{\eta R 12}^{(3)} \gamma_{5}\right) s \frac{1}{M_{\eta R}^{2(3)}} \bar{d}\left(A_{\eta R 12}^{(3)}+B_{\eta R 12}^{(3)} \gamma_{5}\right) s \\
& -\frac{1}{2} \bar{d}\left(A_{P G 12}+B_{P G 12} \gamma_{5}\right) s \frac{1}{M_{P G}^{2}} \bar{d}\left(A_{P G 12}+B_{P G 12} \gamma_{5}\right) s
\end{aligned}
$$

The eight Higgs squared masses appearing in Eq. (8b) that carry superscripts $( \pm)$ are equal in pairs,

$$
M_{\xi R}^{2(+)}=M_{\xi I}^{2(-)}, \quad M_{\xi R}^{2(-)}=M_{\xi I}^{2(+)}, \quad \xi=\phi, \eta
$$


Although they (as well as $M_{\eta R}^{2(3)}$ ) are given in terms of Lagrangian parameters by Eq. (46b) and Table II of [1], we will not use these expressions, but rather will treat the Higgs masses that are independent, after taking account of Eq. (8c), directly as parameters to be bounded.

The subscripts 12 (or 21) in Eq. (8a) indicate the row 1 to column 2 (or row 2 to column 1) matrix element of the corresponding $2 \times 2$ matrix expressions for the $A$ and $B$ coefficients, which we now give. Because CP invariance of the Lagrangian for the six-Higgs doublet model implies that the Yukawa couplings appearing in Eq. (8a) are real, the $A$ coefficients appearing in Eq. (8a) are related to the $B$ coefficients as follows,

$$
\begin{aligned}
A_{\phi, \eta R}^{( \pm)} & =-i B_{\phi, \eta I}^{( \pm)} \\
A_{\phi, \eta I}^{( \pm)} & =i B_{\phi, \eta R}^{( \pm)} \\
A_{\eta R}^{(3)} & =i \frac{\Omega_{A V}}{\Omega_{\phi}} B_{P G}, \\
A_{P G} & =-i \frac{\Omega_{\phi}}{\Omega_{A V}} B_{\eta R}^{(3)} \\
\Omega_{A V} & \equiv\left(\Omega_{\phi}^{2}+\Omega_{\eta}^{2}\right)^{\frac{1}{2}}
\end{aligned}
$$

Defining

$$
M_{2 \times 2}^{(1)}=\left(\begin{array}{ll}
1 & 0 \\
0 & 0
\end{array}\right), \quad M_{2 \times 2}^{(2)}=\left(\begin{array}{ll}
0 & 0 \\
0 & 1
\end{array}\right), \quad \rho_{3}=\left(\begin{array}{cc}
1 & 0 \\
0 & -1
\end{array}\right)
$$

the matrices $B_{\phi R, I}^{( \pm)}$are given by

$$
\begin{aligned}
& B_{\phi R}^{(+)}=\frac{\sqrt{ } 3}{4} g_{\phi}^{d}\left(\hat{V}_{L}^{d} \hat{V}_{R}^{d \dagger}-\hat{V}_{R}^{d} \hat{V}_{L}^{d \dagger}\right), \\
& B_{\phi R}^{(-)}=\frac{\sqrt{ } 3}{4} g_{\phi}^{d}\left(\hat{V}_{L}^{d} \rho_{3} \hat{V}_{R}^{d \dagger}-\hat{V}_{R}^{d} \rho_{3} \hat{V}_{L}^{d \dagger}\right), \\
& B_{\phi I}^{(+)}=\frac{\sqrt{ } 3}{4} g_{\phi}^{d} i\left(\hat{V}_{L}^{d} \hat{V}_{R}^{d \dagger}+\hat{V}_{R}^{d} \hat{V}_{L}^{d \dagger}\right), \\
& B_{\phi I}^{(-)}=\frac{\sqrt{ } 3}{4} g_{\phi}^{d} i\left(\hat{V}_{L}^{d} \rho_{3} \hat{V}_{R}^{d \dagger}+\hat{V}_{R}^{d} \rho_{3} \hat{V}_{L}^{d \dagger}\right),
\end{aligned}
$$


the matrices $B_{\eta R, I}^{( \pm)}$are given by

$$
\begin{aligned}
& B_{\eta R}^{(+)}=-B_{\eta R}^{(-)}=\frac{\sqrt{ } 3}{4} g_{\eta}^{d}\left[\exp (i \theta) \hat{V}_{L}^{d} M_{2 \times 2}^{(1)} \hat{V}_{R}^{d \dagger}-\exp (-i \theta) \hat{V}_{R}^{d} M_{2 \times 2}^{(1)} \hat{V}_{L}^{d \dagger}\right] \\
& B_{\eta I}^{(+)}=-B_{\eta I}^{(-)}=\frac{\sqrt{ } 3}{4} g_{\eta}^{d} i\left[\exp (i \theta) \hat{V}_{L}^{d} M_{2 \times 2}^{(1)} \hat{V}_{R}^{d \dagger}+\exp (-i \theta) \hat{V}_{R}^{d} M_{2 \times 2}^{(1)} \hat{V}_{L}^{d \dagger}\right]
\end{aligned}
$$

and the matrices $B_{\eta R}^{(3)}$ and $B_{P G}$ are given by

$$
\begin{aligned}
B_{\eta R}^{(3)} & =\frac{\sqrt{ } 6}{4} g_{\eta}^{d}\left[\exp (i \theta) \hat{V}_{L}^{d} M_{2 \times 2}^{(2)} \hat{V}_{R}^{d \dagger}-\exp (-i \theta) \hat{V}_{R}^{d} M_{2 \times 2}^{(2)} \hat{V}_{L}^{d \dagger}\right] \\
B_{P G} & =-\frac{\sqrt{ } 6}{4} i \frac{\Omega_{\phi}}{\Omega_{A V}} g_{\eta}^{d}\left[\exp (i \theta) \hat{V}_{L}^{d} M_{2 \times 2}^{(2)} \hat{V}_{R}^{d \dagger}+\exp (-i \theta) \hat{V}_{R}^{d} M_{2 \times 2}^{(2)} \hat{V}_{L}^{d \dagger}\right]
\end{aligned}
$$

In the above formulas, $\theta$ is the overall phase rotation angle between the $\phi$ and $\eta$ Higgs expectations introduced in Eq. (21) of [1].

Taking the $K$ to $\bar{K}$ matrix element of Eq. (8b), we get

$$
\left\langle K\left|\mathcal{H}_{\text {eff }}^{\Delta S=2}\right| \bar{K}\right\rangle=-\frac{1}{2} S_{A}^{d}\left\langle K\left|(\bar{d} s)^{2}\right| \bar{K}\right\rangle-\frac{1}{2} S_{B}^{d}\left\langle K\left|\left(\bar{d} \gamma_{5} s\right)^{2}\right| \bar{K}\right\rangle
$$

with

$$
\begin{aligned}
& S_{A}^{d}=\left(\sum_{\xi=\phi, \eta} \sum_{p= \pm} \sum_{F=R, I} \frac{\left(A_{\xi F 12}^{(p)}\right)^{2}}{M_{\xi F}^{2(p)}}\right)+\frac{\left(A_{\eta R 12}^{(3)}\right)^{2}}{M_{\eta R}^{2(3)}}+\frac{A_{P G 12}^{2}}{M_{P G}^{2}} \\
& S_{B}^{d}=\left(\sum_{\xi=\phi, \eta} \sum_{p= \pm} \sum_{F=R, I} \frac{\left(B_{\xi F 12}^{(p)}\right)^{2}}{M_{\xi F}^{2(p)}}\right)+\frac{\left(B_{\eta R}^{(3)}\right)^{2}}{M_{\eta R}^{2(3)}}+\frac{B_{P G 12}^{2}}{M_{P G}^{2}}
\end{aligned}
$$

The corresponding formulas for the Higgs exchange contribution to the $D$ to $\bar{D}$ transition amplitude are obtained by replacing $K$ by $\bar{D}, d$ by $u$, and $s$ by $c$ in the above formulas, and replacing the explicit factors of $i$ by $-i$ in Eqs. (9b, c, d), with the latter substitution reflecting the fact that the up sector Yukawa couplings involve the charge conjugates of the Higgs fields.

Substituting now the explicit forms given in Eqs. (7a-e) for the matrices $\hat{V}_{R, L}^{d, u}$, we get formulas for the sums $S_{A}^{d}, S_{B}^{d}$ that determine the Higgs exchange contribution to the $\bar{K}$ to $K$ transition amplitude, and for the corresponding sums $S_{A}^{u}$ and $S_{B}^{u}$ that contribute to the 
$D$ to $\bar{D}$ transition amplitude. With an eye to how these formulas will be used in Sec. IV, we write them as

$$
\begin{aligned}
& S_{A}^{d}=s_{d}^{2} c_{d}^{2} \exp \left(i \Phi_{d}\right) P_{A}^{d}, \\
& S_{B}^{d}=-s_{d}^{2} c_{d}^{2} \exp \left(i \Phi_{d}\right) P_{B}^{d}, \\
& S_{A}^{u}=s_{u}^{2} c_{u}^{2} \exp \left(i \Phi_{u}\right) P_{A}^{u}, \\
& S_{B}^{u}=-s_{u}^{2} c_{u}^{2} \exp \left(i \Phi_{u}\right) P_{B}^{u},
\end{aligned}
$$

with the positive real quantities $P_{A, B}^{d, u}$ given by

$$
\begin{aligned}
P_{A}^{d} & =3\left[\left(g_{\phi}^{d}\right)^{2}\left(\frac{\sin ^{2} Y_{d}}{M_{\phi R}^{2(+)}}+\frac{\cos ^{2} Y_{d}}{M_{\phi R}^{2(-)}}\right)\right. \\
& \left.+\frac{1}{4}\left(g_{\eta}^{d}\right)^{2}\left(\frac{1}{M_{\eta R}^{2(+)}}+\frac{1}{M_{\eta R}^{2(-)}}\right)+\frac{1}{2}\left(g_{\eta}^{d}\right)^{2}\left(\frac{\cos ^{2} X_{d}}{M_{\eta}^{2(3)}}+\frac{\Omega_{\phi}^{2}}{\Omega_{A V}^{2}} \frac{\sin ^{2} X_{d}}{M_{P G}^{2}}\right)\right], \\
P_{B}^{d} & =3\left[\left(g_{\phi}^{d}\right)^{2}\left(\frac{\cos ^{2} Y_{d}}{M_{\phi R}^{2(+)}}+\frac{\sin ^{2} Y_{d}}{M_{\phi R}^{2(-)}}\right)\right. \\
& \left.+\frac{1}{4}\left(g_{\eta}^{d}\right)^{2}\left(\frac{1}{M_{\eta R}^{2(+)}}+\frac{1}{M_{\eta R}^{2(-)}}\right)+\frac{1}{2}\left(g_{\eta}^{d}\right)^{2}\left(\frac{\sin ^{2} X_{d}}{M_{\eta}^{2(3)}}+\frac{\Omega_{\phi}^{2}}{\Omega_{A V}^{2}} \frac{\cos ^{2} X_{d}}{M_{P G}^{2}}\right)\right], \\
P_{A}^{u} & =3\left[\left(g_{\phi}^{u}\right)^{2}\left(\frac{\sin ^{2} Y_{u}}{M_{\phi R}^{2(+)}}+\frac{\cos ^{2} Y_{u}}{M_{\phi R}^{2(-)}}\right)\right. \\
& \left.+\frac{1}{4}\left(g_{\eta}^{u}\right)^{2}\left(\frac{1}{M_{\eta R}^{2(+)}}+\frac{1}{M_{\eta R}^{2(-)}}\right)+\frac{1}{2}\left(g_{\eta}^{u}\right)^{2}\left(\frac{\cos ^{2} X_{u}}{M_{\eta}^{2(3)}}+\frac{\Omega_{\phi}^{2}}{\Omega_{A V}^{2}} \frac{\sin ^{2} X_{u}}{M_{P G}^{2}}\right)\right], \\
P_{B}^{u} & =3\left[\left(g_{\phi}^{u}\right)^{2}\left(\frac{\cos ^{2} Y_{u}}{M_{\phi R}^{2(+)}}+\frac{\sin ^{2} Y_{u}}{M_{\phi R}^{2(-)}}\right)\right. \\
& \left.+\frac{1}{4}\left(g_{\eta}^{u}\right)^{2}\left(\frac{1}{M_{\eta R}^{2(+)}}+\frac{1}{M_{\eta R}^{2(-)}}\right)+\frac{1}{2}\left(g_{\eta}^{u}\right)^{2}\left(\frac{\sin ^{2} X_{u}}{M_{\eta}^{2(3)}}+\frac{\Omega_{\phi}^{2}}{\Omega_{A V}^{2}} \frac{\cos ^{2} X_{u}}{M_{P G}^{2}}\right)\right] .
\end{aligned}
$$

The mixing and phase angles appearing in Eqs. (11a, b) are given in terms of the various 
phase angles defined above by

$$
\begin{aligned}
& Y_{d}=\frac{1}{2}\left(\theta_{1}^{d}+\theta_{2}^{d}\right), \\
& X_{d}=\theta+\frac{1}{2}\left(\phi_{R}^{d}-\phi_{L}^{d}\right)-\frac{1}{2}\left(\theta_{1}^{d}+\theta_{2}^{d}\right), \\
& \Phi_{d}=\theta_{1}^{d}-\theta_{2}^{d}-2 \theta_{11}+2 \theta_{21}-\left(\phi_{R}^{d}+\phi_{L}^{d}\right), \\
& Y_{u}=\frac{1}{2}\left(\theta_{1}^{u}+\theta_{2}^{u}\right), \\
& X_{u}=-\theta+\frac{1}{2}\left(\phi_{R}^{u}-\phi_{L}^{u}\right)-\frac{1}{2}\left(\theta_{1}^{u}+\theta_{2}^{u}\right), \\
& \Phi_{u}=\theta_{1}^{u}-\theta_{2}^{u}-2 \theta_{22}+2 \theta_{21}-\left(\phi_{R}^{u}+\phi_{L}^{u}\right),
\end{aligned}
$$

As a check on our phase conventions, we note that when the model is CP conserving, which implies [1] the additional condition $\sigma_{22}^{f}=\sigma_{11}^{f *}$, then Eq. (4d) reduces to $\Theta=\pi / 4$, and the following relations hold (modulo $\pi$ ),

$$
\begin{aligned}
& \theta_{1}^{d, u}=-\theta_{2}^{d, u}=\arg \sigma_{11}^{d, u}, \\
& \phi_{L}^{d, u}=-\arg \sigma_{12}^{d, u}-\arg \sigma_{11}^{d, u}, \\
& \phi_{R}^{d, u}=-\arg \sigma_{12}^{d, u}+\arg \sigma_{11}^{d, u}, \\
& 2 \theta_{11}=-2 \theta_{22}=\phi_{L}^{u}-\phi_{L}^{d}, \\
& 2 \theta_{12}=-2 \theta_{21}=-\phi_{L}^{u}-\phi_{L}^{d} .
\end{aligned}
$$

When substituted into Eq. (12a), these relations imply the vanishing (modulo $\pi$ ) of the phases $\Phi_{d}$ and $\Phi_{u}$. Consequently, with the phase conventions used in this paper, the imaginary parts of the Higgs exchange contributions to $K-\bar{K}$ and $\bar{D}-D$ mixing are a direct measure of the $\mathrm{CP}$ violating contributions to these amplitudes.

\section{Higgs Mass Bounds}

We proceed now to derive bounds on the Higgs masses in the six-Higgs doublet model. Let $\Delta M_{K}^{\text {obs }}$ and $\Delta M_{D}^{\text {obs }}$ be respectively the measured value of the $K_{1}-K_{2}$ mass 
difference and the experimental upper bound on the $D_{1}-D_{2}$ mass difference. Since it is reasonable to expect these to set upper limits on possible Higgs contributions to these mass differences, given respectively by [5]

$$
\begin{gathered}
\left|\Delta M_{K_{1}-K_{2}}^{\mathrm{Higgs}}\right|=M_{K}^{-1}\left|\left\langle K\left|\mathcal{H}_{\text {eff }}^{\Delta S=2}\right| \bar{K}\right\rangle\right|, \\
\left|\Delta M_{D_{1}-D_{2}}^{\mathrm{Higgs}}\right|=M_{D}^{-1}\left|\left\langle\bar{D}\left|\mathcal{H}_{\text {eff }}^{\Delta C=2}\right| D\right\rangle\right|,
\end{gathered}
$$

we get the basic inequalities

$$
\begin{gathered}
\Delta M_{K}^{\mathrm{obs}} \geq M_{K}^{-1}\left|\left\langle K\left|\mathcal{H}_{\text {eff }}^{\Delta S=2}\right| \bar{K}\right\rangle\right|, \\
\Delta M_{D}^{\mathrm{obs}} \geq M_{D}^{-1}\left|\left\langle\bar{D}\left|\mathcal{H}_{\text {eff }}^{\Delta C=2}\right| D\right\rangle\right| .
\end{gathered}
$$

These inequalities will be used in this section, both independently and in combination, to derive a number of useful bounds on the Higgs masses.

We begin by rewriting Eqs. (10a, b) and (11a, b) so as to exhibit the features that play a role in our various inequalities. Let us define $p_{K}$ and $p_{D}$ as the negatives of the ratios of the scalar to pseudoscalar matrix elements appearing in Eq. (10a) and in its D meson analog,

$$
\begin{aligned}
& p_{K}=-\frac{\left\langle K\left|(\bar{d} s)^{2}\right| \bar{K}\right\rangle}{\left\langle K\left|\left(\bar{d} \gamma_{5} s\right)^{2}\right| \bar{K}\right\rangle}, \\
& p_{D}=-\frac{\left\langle\bar{D}\left|(\bar{u} c)^{2}\right| D\right\rangle}{\left\langle\bar{D}\left|\left(\bar{u} \gamma_{5} c\right)^{2}\right| D\right\rangle} .
\end{aligned}
$$

According to calculations of $p_{K}$ and $p_{D}$ by the vacuum insertion method $[6,7]$ and the MIT bag model [6], they are positive and small (roughly of order 0.1 in magnitude). The ratio $p_{K}$ can also be extracted from lattice calculations that have been performed [7] for kaon matrix elements, giving the result $p_{K}=0.30 \pm 0.05$, again of positive sign. Although a similar lattice calculation is not yet available for the $D$ system, we will assume that this follows the same pattern as observed in the $K$ system, and that $p_{D}$ (as suggested by the vacuum saturation and bag model calculations) is positive. Substituting Eqs. (10a), (11a), and (14a) into Eq. (13b), our two basic inequalities now take the form 


$$
\begin{aligned}
& M_{K} \Delta M_{K}^{\mathrm{obs}} \geq \frac{1}{2}\left|\left\langle K\left|\left(\bar{d} \gamma_{5} s\right)^{2}\right| \bar{K}\right\rangle\right| s_{d}^{2} c_{d}^{2}\left|P_{B}^{d}+p_{K} P_{A}^{d}\right| \\
& M_{D} \Delta M_{D}^{\mathrm{obs}} \geq \frac{1}{2}\left|\left\langle\bar{D}\left|\left(\bar{u} \gamma_{5} c\right)^{2}\right| D\right\rangle\right| s_{u}^{2} c_{u}^{2}\left|P_{B}^{u}+p_{D} P_{A}^{u}\right|
\end{aligned}
$$

with $P_{B}^{d, u}+p_{K, D} P_{A}^{d, u}$ both sums of positive terms. Introducing the definitions

$$
\begin{aligned}
& E_{K}=\frac{2 M_{K} \Delta M_{K}^{\mathrm{obs}}}{s_{12}^{2} c_{12}^{2}\left|\left\langle K\left|\left(\bar{d} \gamma_{5} s\right)^{2}\right| \bar{K}\right\rangle\right|} \\
& E_{D}=\frac{2 M_{D} \Delta M_{D}^{\mathrm{obs}}}{s_{12}^{2} c_{12}^{2}\left|\left\langle\bar{D}\left|\left(\bar{u} \gamma_{5} c\right)^{2}\right| D\right\rangle\right|}
\end{aligned}
$$

we rewrite the inequalities of Eq. (14b) as

$$
\begin{aligned}
& \left(\frac{E_{K}}{\left|P_{B}^{d}+p_{K} P_{A}^{d}\right|}\right)^{\frac{1}{2}} \geq \frac{\left|s_{d} c_{d}\right|}{s_{12} c_{12}}, \\
& \left(\frac{E_{D}}{\left|P_{B}^{u}+p_{D} P_{A}^{u}\right|}\right)^{\frac{1}{2}} \geq \frac{\left|s_{u} c_{u}\right|}{s_{12} c_{12}}
\end{aligned} .
$$

Although Eqs. (14d) are relevant for the numerical fits of Sec. VI, where the products $\left|s_{d} c_{d}\right|$ and $\left|s_{u} c_{u}\right|$ are known, they cannot be used to give fit-independent bounds on the Higgs masses, because either $\left|s_{d} c_{d}\right|$ or $\left|s_{u} c_{u}\right|$ can vanish. However, we shall now show that the sum $\left|s_{d} c_{d}\right|+\left|s_{u} c_{u}\right|$ is bounded below by CKM matrix elements, permitting us to extract a useful inequality by combining the $K$ meson and $D$ meson flavor changing neutral current constraints. To see this, we substitute Eq. (4b) for $V_{L}^{d, u}$, together with the definitions of Eq. (7e), into Eq. (6b) for the adjoint of the unrephased CKM matrix, and take absolute values of the matrix elements on the first row, giving

$$
\begin{aligned}
& s_{12}=\left|c_{u} s_{d} \exp \left(-i \phi_{L}^{d}\right)-s_{u} c_{d} \exp \left(-i \phi_{L}^{u}\right)\right| \leq\left|c_{u} s_{d}\right|+\left|s_{u} c_{d}\right| \quad, \\
& c_{12}=\left|c_{u} c_{d}+\exp \left(i \phi_{L}^{u}-i \phi_{L}^{d}\right) s_{u} s_{d}\right| \leq\left|c_{u} c_{d}\right|+\left|s_{u} s_{d}\right| .
\end{aligned}
$$

Multiplying these inequalities, we get

$$
s_{12} c_{12} \leq\left|c_{d} s_{d}\right|\left(s_{u}^{2}+c_{u}^{2}\right)+\left|c_{u} s_{u}\right|\left(s_{d}^{2}+c_{d}^{2}\right)=\left|c_{d} s_{d}\right|+\left|c_{u} s_{u}\right|
$$


giving the needed lower bound. Adding the two inequalities in Eq. (14d), and using Eq. (15b), we get the master inequality

$$
\left(\frac{E_{K}}{\left|P_{B}^{d}+p_{K} P_{A}^{d}\right|}\right)^{\frac{1}{2}}+\left(\frac{E_{D}}{\left|P_{B}^{u}+p_{D} P_{A}^{u}\right|}\right)^{\frac{1}{2}} \geq 1 .
$$

Since all terms in the denominators $\left|P_{B}^{d, u}+p_{K, D} P_{A}^{d, u}\right|$ are positive, deleting any of these terms serves to make the left hand side of Eq. (16) larger, giving a number of simpler subsidiary inequalities that are consequences of the master inequality. Specifically, if we delete all terms in both denominators that do not refer to a given Higgs mass (i.e., if we set all of the other Higgs masses equal to infinity), we get a lower bound for the Higgs mass that we have retained; performing this in succession for the six Higgs masses we get the following inequalities,

$$
\begin{aligned}
& M_{\phi R}^{(+)} \geq\left\{\left[\frac{E_{K}}{3\left(g_{\phi}^{d}\right)^{2}\left(\cos ^{2} Y_{d}+p_{K} \sin ^{2} Y_{d}\right)}\right]^{\frac{1}{2}}+\left[\frac{E_{K}}{3\left(g_{\phi}^{u}\right)^{2}\left(\cos ^{2} Y_{u}+p_{D} \sin ^{2} Y_{u}\right)}\right]^{\frac{1}{2}}\right\}^{-1}, \\
& M_{\phi R}^{(-)} \geq\left\{\left[\frac{E_{D}}{3\left(g_{\phi}^{d}\right)^{2}\left(\sin ^{2} Y_{d}+p_{K} \cos ^{2} Y_{d}\right)}+\left[\frac{1}{3\left(g_{\phi}^{u}\right)^{2}\left(\sin ^{2} Y_{u}+p_{D} \cos ^{2} Y_{u}\right)}\right]^{\frac{1}{2}}\right\}^{-1},\right. \\
& \left.M_{\eta R}^{( \pm)} \geq\left\{\left[\frac{4 E_{K}}{3\left(g_{\eta}^{d}\right)^{2}\left(1+p_{K}\right)}\right]^{\frac{1}{2}}+\left[\frac{4 E_{D}}{3\left(g_{\eta}^{u}\right)^{2}\left(1+p_{D}\right)}\right]^{\frac{1}{2}}\right\}^{-1},\left[\frac{2 E_{D}}{3\left(g_{\eta}^{u}\right)^{2}\left(\sin ^{2} X_{u}+p_{D} \cos ^{2} X_{u}\right)}\right]^{\frac{1}{2}}\right\}^{-1}, \\
& M_{\eta R}^{(3)} \geq\left\{\left[\frac{1}{3\left(g_{\eta}^{d}\right)^{2}\left(\sin ^{2} X_{d}+p_{K} \cos ^{2} X_{d}\right)}\right]^{\frac{1}{2}}\left[\frac{2 E_{D} \Omega_{A V}^{2}}{2 E_{K} \Omega_{A V}^{2}}\right]^{\frac{1}{2}}+\left[\frac{\sin ^{2}}{3\left(g_{\eta}^{u}\right)^{2} \Omega_{\phi}^{2}\left(\cos ^{2} X_{u}+p_{D} \sin ^{2} X_{u}\right)}\right]^{-1} .\right.
\end{aligned}
$$

Applying the same procedure of successive deletion of denominator terms to the inequalities of Eq. (14d), we get a set of analogous inequalities [which, by use of Eq. (15b), imply those 
of Eq. (17a)] that will be used in the numerical work of Sec. VI,

$$
\begin{aligned}
& M_{\phi R}^{(+)} \geq \max \left\{r_{d}\left[\frac{E_{K}}{3\left(g_{\phi}^{d}\right)^{2}\left(\cos ^{2} Y_{d}+p_{K} \sin ^{2} Y_{d}\right)}\right]^{-\frac{1}{2}}, r_{u}\left[\frac{E_{D}}{3\left(g_{\phi}^{u}\right)^{2}\left(\cos ^{2} Y_{u}+p_{D} \sin ^{2} Y_{u}\right)}\right]^{-\frac{1}{2}}\right\}, \\
& M_{\phi R}^{(-)} \geq \max \left\{r_{d}\left[\frac{E_{K}}{3\left(g_{\phi}^{d}\right)^{2}\left(\sin ^{2} Y_{d}+p_{K} \cos ^{2} Y_{d}\right)}\right]^{-\frac{1}{2}}, r_{u}\left[\frac{E_{D}}{3\left(g_{\phi}^{u}\right)^{2}\left(\sin ^{2} Y_{u}+p_{D} \cos ^{2} Y_{u}\right)}\right]^{-\frac{1}{2}}\right\}, \\
& M_{\eta R}^{( \pm)} \geq \max \left\{r_{d}\left[\frac{4 E_{K}}{3\left(g_{\eta}^{d}\right)^{2}\left(1+p_{K}\right)}\right]^{-\frac{1}{2}}, r_{u}\left[\frac{4 E_{D}}{3\left(g_{\eta}^{u}\right)^{2}\left(1+p_{D}\right)}\right]^{-\frac{1}{2}}\right\} \\
& M_{\eta R}^{(3)} \geq \max \left\{r_{d}\left[\frac{2 E_{K}}{3\left(g_{\eta}^{d}\right)^{2}\left(\sin ^{2} X_{d}+p_{K} \cos ^{2} X_{d}\right)}\right]^{-\frac{1}{2}}, r_{u}\left[\frac{2 E_{D}}{3\left(g_{\eta}^{u}\right)^{2}\left(\sin ^{2} X_{u}+p_{D} \cos ^{2} X_{u}\right)}\right]^{-\frac{1}{2}}\right\}, \\
& M_{P G} \geq \max \left\{r_{d}\left[\frac{2 E_{K} \Omega_{A V}^{2}}{3\left(g_{\eta}^{d}\right)^{2} \Omega_{\phi}^{2}\left(\cos ^{2} X_{d}+p_{K} \sin ^{2} X_{d}\right)}\right]^{-\frac{1}{2}}, r_{u}\left[\frac{2 E_{D} \Omega_{A V}^{2}}{3\left(g_{\eta}^{u}\right)^{2} \Omega_{\phi}^{2}\left(\cos ^{2} X_{u}+p_{D} \sin ^{2} X_{u}\right)}\right]^{-\frac{1}{2}}\right\} \text {, } \\
& r_{d} \equiv \frac{\left|s_{d} c_{d}\right|}{s_{12} c_{12}}, \quad r_{u} \equiv \frac{\left|s_{u} c_{u}\right|}{s_{12} c_{12}} .
\end{aligned}
$$

The bounds in Eq. (17a) still depend on the mixing angles $X_{d, u}$ and $Y_{d, u}$ defined in Eq. (12a); a set of (necessarily weaker) bounds that do not depend on these angles is obtained by using the inequalities, valid for $p \leq 1$,

$$
\begin{aligned}
& \cos ^{2} Z+p \sin ^{2} Z=(1-p) \cos ^{2} Z+p \geq p, \\
& \sin ^{2} Z+p \cos ^{2} Z=(1-p) \sin ^{2} Z+p \geq p,
\end{aligned}
$$

giving (for $\left.p_{K, D} \leq 1\right)$ the inequalities

$$
\begin{aligned}
& M_{\phi R}^{( \pm)} \geq\left\{\left[\frac{E_{K}}{3\left(g_{\phi}^{d}\right)^{2} p_{K}}\right]^{\frac{1}{2}}+\left[\frac{E_{D}}{3\left(g_{\phi}^{u}\right)^{2} p_{D}}\right]^{\frac{1}{2}}\right\}^{-1}, \\
& M_{\eta R}^{( \pm)} \geq\left\{\left[\frac{4 E_{K}}{3\left(g_{\eta}^{d}\right)^{2}\left(1+p_{K}\right)}\right]^{\frac{1}{2}}+\left[\frac{4 E_{D}}{3\left(g_{\eta}^{u}\right)^{2}\left(1+p_{D}\right)}\right]^{\frac{1}{2}}\right\}^{-1}, \\
& M_{\eta R}^{(3)} \geq\left\{\left[\frac{2 E_{K}}{3\left(g_{\eta}^{d}\right)^{2} p_{K}}\right]^{\frac{1}{2}}+\left[\frac{2 E_{D}}{3\left(g_{\eta}^{u}\right)^{2} p_{D}}\right]^{\frac{1}{2}}\right\}^{-1}, \\
& M_{P G} \geq\left\{\left[\frac{2 E_{K} \Omega_{A V}^{2}}{3\left(g_{\eta}^{d}\right)^{2} \Omega_{\phi}^{2} p_{K}}\right]^{\frac{1}{2}}+\left[\frac{2 E_{D} \Omega_{A V}^{2}}{3\left(g_{\eta}^{u}\right)^{2} \Omega_{\phi}^{2} p_{D}}\right]^{\frac{1}{2}}\right\}^{-1} .
\end{aligned}
$$


Equations (17a, b) and (18b) are our final bounds for the Higgs masses in the six-Higgs doublet model.

To obtain numerical values from the bounds of Eq. (18b), we first need to evaluate the ratios $E_{K, D}$ defined in Eq. (14c). For $E_{K}$ we use the measured value [8] $\Delta M_{K}^{\text {obs }}=3.49 \times$ $10^{-12} \mathrm{MeV}$, together with $M_{K}=497.7 \mathrm{MeV}, M_{d}=6 \mathrm{MeV}, M_{s}=115 \mathrm{MeV}, f_{K}=160 \mathrm{MeV}$, $c_{12}=0.975, s_{12}=0.221$, and the lattice evaluation [7]

$$
\left|\left\langle K\left|\left(\bar{d} \gamma_{5} s\right)^{2}\right| \bar{K}\right\rangle\right|=1.58\left(\frac{M_{K}}{M_{s}+M_{d}}\right)^{2} M_{K}^{2} f_{K}^{2} \quad,
$$

to give

$$
E_{K}=0.44 \times 10^{-12}(\mathrm{GeV})^{-2}
$$

For $E_{D}$, we use the experimental upper bound [8] $\Delta M_{D}^{\text {obs }}=1.58 \times 10^{-10} \mathrm{MeV}$, together with $M_{D}=1865 \mathrm{MeV}, M_{u}=3.25 \mathrm{MeV}, M_{c}=1.25 \mathrm{GeV}$, the lattice calculation [9] value $f_{D} \simeq 1.2 f_{K}$, and the vacuum saturation approximation formula [7]

$$
\frac{\mid\left\langle K\left|\left(\bar{d} \gamma_{5} s\right)^{2}\right| \bar{K}\right\rangle}{\left|\left\langle\bar{D}\left|\left(\bar{u} \gamma_{5} c\right)^{2}\right| D\right\rangle\right|}=\frac{f_{K}^{2} M_{K}^{2}\left[11 M_{K}^{2}\left(M_{s}+M_{d}\right)^{-2}+1\right]}{f_{D}^{2} M_{D}^{2}\left[11 M_{D}^{2}\left(M_{c}+M_{u}\right)^{-2}+1\right]},
$$

to give

$$
E_{D}=27 \times 10^{-12}(\mathrm{GeV})^{-2}
$$

Since the scalar to pseudoscalar ratio $p_{D}$ has not yet been computed on the lattice, we will assume that $p_{D}=p_{K}=0.3$ in evaluating Eqs. (18b).

To complete the computation of Higgs mass bounds, we need the values of the various Yukawa couplings appearing in Eqs. (18b). Here some assumptions about how the first and second family masses are generated are needed. If, following [1], we assume that the $\phi$ Higgs expectations generate the third family masses, the $\eta$ Higgs expectations generate the second 
family masses, while cyclic asymmetries in the Yukawa couplings are responsible for the first family masses, then we get from Eq. (32b) of [1] the formulas

$$
\begin{aligned}
& g_{\phi}^{u}=\frac{M_{t}}{3 \Omega_{\phi}}, \quad g_{\phi}^{d}=\frac{M_{b}}{3 \Omega_{\phi}}, \\
& g_{\eta}^{u}=\frac{M_{c}}{3 \Omega_{\eta}}, \quad g_{\eta}^{d}=\frac{M_{s}}{3 \Omega_{\eta}} .
\end{aligned}
$$

If we now assume equal $\Omega_{\phi}$ and $\Omega_{\eta}$, so that $\Omega_{\phi}=\Omega_{\eta}=71 \mathrm{GeV}$, we get the numerical values

$$
\begin{aligned}
& g_{\phi}^{u}=0.82, \quad g_{\phi}^{d}=0.020, \\
& g_{\eta}^{u}=0.0059, \quad g_{\eta}^{d}=0.00054,
\end{aligned}
$$

giving the Higgs mass lower bounds

$$
\begin{aligned}
& M_{\phi R}^{( \pm)} \geq 24 \mathrm{TeV}, \\
& M_{\eta R}^{( \pm)} \geq 470 \mathrm{GeV}, \\
& M_{\eta R}^{(3)} \geq 320 \mathrm{GeV}, \\
& M_{P G} \geq 220 \mathrm{GeV} .
\end{aligned}
$$

An alternative possibility, discussed in the next section, is that the second family masses are generated by cyclic asymmetries in the $\phi$ Yukawa couplings, with the first family masses generated by the $\eta$ Higgs expectations. In this case, the second line of Eq. (20a) is replaced by

$$
g_{\eta}^{u} \simeq \frac{M_{u}}{1.5 \Omega_{\eta}}, \quad g_{\eta}^{d} \simeq \frac{M_{d}}{1.5 \Omega_{\eta}}
$$

giving (for $\Omega_{\eta}=\Omega_{\phi}$ ) the numerical values $g_{\eta}^{u}=3.1 \times 10^{-5}, \quad g_{\eta}^{d}=5.7 \times 10^{-5}$, which imply the much weaker $\eta$ and pseudo Goldstone Higgs mass bounds

$$
\begin{aligned}
& M_{\eta R}^{( \pm)} \geq 5.5 \mathrm{GeV}, \\
& M_{\eta R}^{(3)} \geq 3.7 \mathrm{GeV}, \\
& M_{P G} \geq 2.6 \mathrm{GeV} .
\end{aligned}
$$


Finally, we note that the first inequality of Eq. (18b) also applies to the CP conserving case of the three-Higgs doublet model of [1], for which $\Omega_{\phi}=\sqrt{ } 2 \times 71 \mathrm{GeV}$, so that $g_{\phi}^{d, u}$ are a factor of $\sqrt{ } 2$ smaller than given in Eq. (20b). This reduces the corresponding bounds of Eq. (18b) by a factor of $\sqrt{ } 2$, giving for the CP-conserving three-Higgs doublet model the Higgs mass lower bounds

$$
M_{\phi R}^{( \pm)} \geq 17 \mathrm{TeV}
$$

In this model there are no $\eta$ Higgs states, and hence no possibility of neutral Higgs states that are not supermassive.

\section{Implications of Requiring Naturally Small First Family Masses}

We saw in the preceding section that the bounds on the $\eta$ and pseudo Goldstone Higgs masses depend on the coupling pattern assumed for the $\eta$ Higgs discrete chiral triplet. In this section we classify possible $\eta$ Higgs coupling patterns, based on a criterion of requiring naturally small first family masses. Referring to Eqs. (1a) and (1b), we see that contributions to the mass matrix in the six-Higgs doublet model are of three distinct types, arising from deviations from cyclic symmetry in the $\phi$ Higgs Yukawa couplings, deviations from cyclic symmetry in the $\phi$ Higgs expectations, and contributions from the weakly coupled $\eta$ Higgs expectations. Since these three contributions are not directly related physically, detailed cancellations between them in the determination of the first family masses are a priori unlikely. Hence as a necessary (but not sufficient) condition for naturally small first family masses, we impose the condition that only one of these three contributions dominates in a leading approximation in which the first family masses are exactly zero.

We begin by noting that the deviations $\delta_{n}^{f}$ from cyclic symmetry in the $\phi$ Higgs 
Yukawa couplings cannot dominate and lead to naturally zero first family masses. Let us suppose that the $\delta_{n}^{f}$ do dominate, and consider first the case in which the model chooses a CP conserving ground state, for which the parameters $\delta_{n}^{f}$ are all real. In this case the magnitudes

$$
\begin{aligned}
& \left|\sigma_{11}^{f}\right| \simeq\left|\delta_{3}^{f}+\bar{\omega} \delta_{2}^{f}+\omega \delta_{1}^{f}\right|, \\
& \left|\sigma_{22}^{f}\right| \simeq\left|\delta_{3}^{f}+\omega \delta_{2}^{f}+\bar{\omega} \delta_{1}^{f}\right|,
\end{aligned}
$$

are equal, and so the first and second family masses are equal. Turning on a CP violation results in complex $\delta_{n}^{f}$ 's, for which the first and second family masses are no longer the same, but clearly a fine tuning of the amount of $\mathrm{CP}$ violation would be needed to achieve zero first family masses. Hence dominance of the Higgs expectation asymmetries $\delta_{n}^{f}$ is not compatible with naturally small first family masses.

We consider next the case in which the contribution $R^{f}$ of the $\eta$ Higgs expectations dominates, which is the scenario assumed in [1]. In this case the $\eta$ Higgs expectations give rise to the second family masses, and the leading approximation to the first family masses is automatically zero, satisfying our criterion for naturally small first family masses. However, a potential problem arises when we examine the structure of the CKM matrix. In the leading approximation in which only $R^{f}$ is retained in the mass matrix, the CKM matrix is unity. To get a nontrivial CKM matrix, we must add back the small perturbations $\delta_{n}^{f}$ and $\mu_{\ell m}^{f}$ in Eq. (1b). According to Eqs. (4a-d), in each flavor channel we then get

$$
\begin{aligned}
A-B & \simeq-\left|\sigma_{22}\right|^{2}, \\
|z| & \simeq\left|\sigma_{12}\right|\left|\sigma_{22}\right|, \\
\Theta & \simeq \frac{1}{2} \tan ^{-1}\left(\frac{2\left|\sigma_{12}\right|}{\left|\sigma_{22}\right|}\right),
\end{aligned}
$$

which by the hypothesis of dominance of $R^{f}$ is much less than unity. Hence, in particular, the up channel quantity $s^{u}=\sin \Theta^{u}$ is much less than unity in magnitude. But referring 
now to the corrected expressions [3] for the CKM elements $s_{13}$ and $s_{23}$, we have

$$
\begin{aligned}
& s_{13}=\left|s_{3}-d_{3}\right| / 3, \quad s_{23}=\left|s_{3}+d_{3}\right| / 3, \\
& s_{3}=c_{u}\left(\sigma_{13}^{d}-\sigma_{13}^{u}\right), \quad d_{3}=s_{u} \exp \left(-i \phi_{L}^{u}\right)\left(\sigma_{23}^{d}-\sigma_{23}^{u}\right),
\end{aligned}
$$

indicating that the spread of $s_{23}$ and $s_{13}$ from their geometric mean is suppressed by the small quantity $s_{u}$. This in turn requires relatively large parameter values $\sigma_{23}$ and/or $\sigma_{13}$ to give a satisfactory fit to the data, contradicting the starting assumption of a dominant $R^{f}$. We shall see evidence for this phenomenon in the next section, where we find Yukawa asymmetries comparable in magnitude to $R^{f}$, and hence substantial fine tuning in achieving small first family masses.

We turn finally to the third case, in which the dominant contributions to the mass matrix come from the asymmetries $\mu_{\ell m}^{f}$ of the $\phi$ Higgs Yukawa couplings. For the leading approximation to the $2 \times 2$ submatrix $m$ of the mass matrix, we then have (suppressing the flavor index $f$ )

$$
m=\frac{1}{3}\left(\begin{array}{ll}
\mu_{11} & \mu_{12} \\
\mu_{21} & \mu_{22}
\end{array}\right)
$$

with only two of the matrix elements in Eq. (25a) independent, since CP invariance of the $\phi$ Higgs Yukawa couplings implies [1] that

$$
\mu_{21}=\mu_{12}^{*}, \quad \mu_{22}=\mu_{11}^{*}
$$

In order for Eq. (25a) to have a zero eigenvalue, we must impose the additional condition

$$
\left|\mu_{12}\right|=\left|\mu_{11}\right| \Leftrightarrow \mu_{12}=\exp (-i \chi) \mu_{11}^{*}
$$

an explanation for which must be sought in higher energy physics determining the Yukawa couplings. Taken together, Eqs. (26a) and (25b) imply that the matrix $m$ takes the rank one 
form

$$
m=\frac{1}{3}\left(\begin{array}{cc}
\mu_{11} & \exp (-i \chi) \mu_{11}^{*} \\
\exp (i \chi) \mu_{11} & \mu_{11}^{*}
\end{array}\right)
$$

with eigenvalues $\left|\kappa_{1}\right|=0$ and $\left|\kappa_{2}\right|=\frac{2}{3}\left|\mu_{11}\right|$, corresponding respectively to the first and second family mass eigenstates. ¿From Eq. (4a), we find that the diagonalizing matrices $V_{L, R}$ are given by Eq. (4b), with

$$
\begin{gathered}
\Theta=\frac{\pi}{4}, \quad \cos \Theta=\sin \Theta=\frac{1}{\sqrt{ } 2}, \\
\phi_{L}=\chi, \quad \phi_{R}=\chi+2 \arg \mu_{11} .
\end{gathered}
$$

Referring to Eq. (15a), we see that the sine of the Cabibbo angle $s_{12}$ is given now by

$$
s_{12}=\frac{1}{2}\left|\exp \left(-i \phi_{L}^{d}\right)-\exp \left(-i \phi_{L}^{u}\right)\right|=\left|\sin \frac{1}{2}\left(\chi^{u}-\chi^{d}\right)\right|
$$

Averaging Eq. (24b) and the analogous expression obtained from the lower left corner of the CKM matrix, we get the following leading order expressions for $s_{13}$ and $s_{23}$,

$$
\begin{aligned}
& s_{13}=\left|s_{3}-d_{3}\right| / 3, \quad s_{23}=\left|s_{3}+d_{3}\right| / 3, \\
& s_{3}=\frac{1}{\sqrt{ } 2}\left(\sigma_{13}^{d}-\sigma_{13}^{u}\right), \quad d_{3}=\frac{1}{\sqrt{ } 2} \exp \left[-\frac{i}{2}\left(\chi^{u}+\chi^{d}\right)\right] \cos \left[\frac{1}{2}\left(\chi^{u}-\chi^{d}\right)\right]\left(\sigma_{23}^{d}-\sigma_{23}^{u}\right),
\end{aligned}
$$

in which the coefficient of $\sigma_{23}^{d}-\sigma_{23}^{u}$ in $d_{3}$ is not now a small parameter.

To complete the analysis of the third case, let us calculate the first family mass eigenvalue. There are four possible contributions to a nonzero first family mass: (i) deviations from the rank one condition of Eq. (26a) on the $2 \times 2$ submatrix $m$ of the mass matrix, (ii) asymmetries in the $\phi$ Higgs expectations $\delta_{n}$, (iii) couplings to the third family through the mass matrix elements $\sigma_{13}, \sigma_{31}$ and $\sigma_{23}, \sigma_{32}$, and (iv) effects of the $\eta$ Higgs expectation term $R$ in Eq. (1b). (We are continuing to suppress the flavor index $f$ when not needed.) The simplest way to calculate the first family mass matrix eigenvalue $\left|\kappa_{1}\right|$ is to evaluate the absolute value of the determinant of the $3 \times 3$ mass matrix $M^{\prime}$ of Eq. (2a), which yields $\left|\kappa_{1}\right|$ 
when divided by the product of the other two eigenvalues, giving to leading order in small quantities,

$$
\left|\kappa_{1}\right| \simeq \frac{\left|\operatorname{det} M^{\prime}\right|}{3\left|\kappa_{2}\right|} \simeq \frac{\left|\operatorname{det}\left(3 M^{(3)}+\sigma\right)\right|}{2\left|\mu_{11}\right|}
$$

To illustrate this in a simple texture model corresponding to the third case, let us assume that the contributions (i) and (ii) above are absent, that is, we assume that the condition of Eq. (26a) is exact and that cyclic asymmetries in the $\phi$ Higgs expectations are negligible. Then the only relevant contributions to the determinant in Eq. (28a) are (iii) and (iv) above, and a simple calculation gives the leading order formula

$$
\left|\kappa_{1}\right| \simeq\left|\frac{3}{2} R-\frac{1}{6}\left[\sigma_{13}^{*}-\exp \left(i \phi_{L}\right) \sigma_{13}\right]\left[\sigma_{31}^{*}-\exp \left(-i \phi_{R}\right) \sigma_{31}\right]\right|
$$

with $\phi_{L, R}$ given in Eq. (27a). When only the $R$ term is retained, substituting Eq. (28b) into Eq. (1a) yields the formulas of Eq. (21a). Within the simplified texture model, we have also calculated the $\mathrm{CP}$ violating angle $\delta_{13}$ appearing in the standard form [8] CKM matrix as a consequence of the $\mathrm{CP}$ violation carried by the $R$ term. After considerable algebra, we find

$$
\delta_{13} \simeq 2 \sqrt{ } 2 \epsilon^{u}\left[\cot \left(\phi_{L}^{u}-\phi_{L}^{d}\right)-\cot \left(\phi_{L}^{u}+2 \theta_{13}\right)\right]-2 \sqrt{ } 2 \epsilon^{d} \csc \left(\phi_{L}^{u}-\phi_{L}^{d}\right) \quad,
$$

with the auxiliary quantities appearing in Eq. (29a) defined by

$$
\theta_{13}=\arg \left(\sigma_{13}^{d}-\sigma_{13}^{u}\right), \quad \epsilon^{u, d}=\frac{-9 \operatorname{Re}\left(\mu_{11}^{u, d} R^{u, d}\right)}{4 \sqrt{ } 2\left|\mu_{11}^{u, d}\right|^{2}}
$$

To complete the specification of the texture model corresponding to the third case, we note that since the $2 \times 2$ diagonalizing submatrices $V_{L, R}$ are maximally mixing in this case, wherever "1st" or "2nd" appears in the Higgs meson fermion family coupling Table II of [1], there now should appear "1st and 2nd", indicating couplings of equal magnitude of 
first family to first family, first family to second family, and second family to second family. We also remark that the rank one condition of Eq. (26a) can be reexpressed as a model for the Yukawa asymmetries $\beta_{\ell m}$, by using the inversion formulas

$$
\begin{aligned}
& \beta_{11}=\frac{2}{9} \operatorname{Re}\left(\mu_{11}+\mu_{12}+\mu_{13}+\mu_{31}\right) \\
& \beta_{12}=\frac{2}{9} \operatorname{Re}\left(\omega \mu_{11}+\bar{\omega} \mu_{12}+\mu_{13}+\omega \mu_{31}\right) \\
& \beta_{13}=\frac{2}{9} \operatorname{Re}\left(\bar{\omega} \mu_{11}+\omega \mu_{12}+\mu_{13}+\bar{\omega} \mu_{31}\right) \\
& \beta_{21}=\frac{2}{9} \operatorname{Re}\left(\omega \mu_{11}+\omega \mu_{12}+\omega \mu_{13}+\mu_{31}\right) \\
& \beta_{22}=\frac{2}{9} \operatorname{Re}\left(\bar{\omega} \mu_{11}+\mu_{12}+\omega \mu_{13}+\omega \mu_{31}\right) \\
& \beta_{23}=\frac{2}{9} \operatorname{Re}\left(\mu_{11}+\bar{\omega} \mu_{12}+\omega \mu_{13}+\bar{\omega} \mu_{31}\right) \\
& \beta_{31}=\frac{2}{9} \operatorname{Re}\left(\bar{\omega} \mu_{11}+\bar{\omega} \mu_{12}+\bar{\omega} \mu_{13}+\mu_{31}\right) \\
& \beta_{32}=\frac{2}{9} \operatorname{Re}\left(\mu_{11}+\omega \mu_{12}+\bar{\omega} \mu_{13}+\omega \mu_{31}\right) \\
& \beta_{33}=\frac{2}{9} \operatorname{Re}\left(\omega \mu_{11}+\mu_{12}+\bar{\omega} \mu_{13}+\bar{\omega} \mu_{31}\right)
\end{aligned}
$$

\section{Some Illustrative Numerical Fits}

We give in this section illustrative numerical fits, obtained by the method of minimizing a "cost function" described in detail in Sec. IX of [1]. The mass and CKM cost functions are as in Eqs. (51b) and (52b) of [1], with the changes that we omit the term $\left(\Omega_{\phi}-\Omega_{\eta}\right)^{2}$, which had little effect on the fits, and take the target values and standard deviations from the latest Particle Data Group [8] compilation. For the parameter cost function, we use Eq. (53a) of [1], with the changes that we omit the summation restrictions excluding the $n=3$ and $m=n=3$ terms, so that all asymmetries are treated symmetrically, and we take the exponent $\epsilon$ as 2 rather than as 1. For the third case of the proceeding section, in which the second family masses arise from Yukawa coupling asymmetries, we also add to 
the parameter cost function a term

$$
\frac{\Omega_{\eta}^{2}}{4}\left[\left(\frac{g_{\eta}^{u}}{M_{u}}\right)^{2}+\left(\frac{g_{\eta}^{d}}{M_{d}}\right)^{2}+\left(\frac{g_{\eta}^{e}}{M_{e}}\right)^{2}\right]
$$

designed to keep the $\eta$ Higgs contributions small, and start the iteration from preliminary parameter values determined with $g_{\eta}^{f}=0, f=u, d, e$. We omit flavor changing neutral current constraints from the cost function, so that there is no fine tuning to attempt to suppress flavor changing neutral current effects; instead we use the inequalities of Eq. (17b), evaluated using the parameters determined by the fitting procedure, to give lower bounds on the Higgs masses that guarantee sufficiently small flavor changing neutral current contributions to $K-\bar{K}$ and $\bar{D}-D$ mixing.

Fitting results for the second case of the proceeding section are given in Table I, obtained with a standard deviation for the Yukawa asymmetries $\beta$ of $\sigma_{\text {parameter }}=0.02$. For this fit, the maximum $|\beta|$ values in the up, down, and electron sectors are $0.032,0.058$, and 0.010 respectively. Although the iteration is started with the $\eta$ Higgs coupling values of Eq. (20b), the converged fit has significantly smaller couplings $g_{\eta}^{u}=0.0031$ and $g_{\eta}^{d}=0.00039$, indicating that the Yukawa asymmetries make substantial contributions to the second family masses. This means that the $\eta$ Higgs contributions do not dominate the Yukawa asymmetries, and thus substantial fine tuning is involved in achieving small first family masses. For the 
fit of Table I, the Higgs mass bounds obtained from Eq. (17b) are

$$
\begin{aligned}
& M_{\phi R}^{(+)} \geq 520 \mathrm{TeV}, \\
& M_{\phi R}^{(-)} \geq 440 \mathrm{TeV}, \\
& M_{\eta R}^{( \pm)} \geq 1.3 \mathrm{TeV}, \\
& M_{\eta R}^{(3)} \geq 1.1 \mathrm{TeV}, \\
& M_{P G} \geq 1.0 \mathrm{TeV} .
\end{aligned}
$$

In Table II we give fitting results for the third case of the preceding section, obtained now with a standard deviation for the Yukawa asymmetries $\beta$ of $\sigma_{\text {parameter }}=0.08$. For this fit, the maximum $|\beta|$ values in the up, down, and electron sectors are $0.042,0.052$, and 0.11 respectively, with the relatively large Yukawa coupling asymmetry needed in the electron sector reflecting the fact that in this sector the ratio of the second family to third family mass is biggest. For the fit of Table II, the Higgs mass bounds obtained from Eq. (17b) are

$$
\begin{aligned}
& M_{\phi R}^{(+)} \geq 370 \mathrm{TeV}, \\
& M_{\phi R}^{(-)} \geq 620 \mathrm{TeV}, \\
& M_{\eta R}^{( \pm)} \geq 210 \mathrm{GeV}, \\
& M_{\eta R}^{(3)} \geq 220 \mathrm{GeV}, \\
& M_{P G} \geq 140 \mathrm{GeV} .
\end{aligned}
$$

To conclude, in order for flavor changing neutral current effects in our models to be sufficiently small, the $\phi$ Higgs masses must be very large, far outside the regime in which conventional perturbative Higgs physics applies (see [10] for a recent review of both perturbative and strongly coupled Higgs models). Our results are consistent with general group theoretic analyses of flavor changing neutral currents in multi-Higgs doublet extensions of the standard model [11], which when applied to our models imply that flavor changing 
neutral currents cannot cancel kinematically, but must be eliminated either by fine tuning (an option we have ruled out by the inequalities of Sec. IV) or by having some very large Higgs masses. From an experimental viewpoint, the most interesting scenario within our models corresponds to the third case discussed in Sec. V, in which the $\eta$ Higgs couplings are small enough that their expectations play an important role only in determining the first family masses and in giving rise to $\mathrm{CP}$ violation. In this case the $\eta$ Higgs and pseudo Goldstone Higgs states are permitted by our mass bounds to be light enough to be seen in experiments at the LHC. The simplest model of this type would be one in which the $\phi$ and $\eta$ Higgs self-interactions have similar structures, with a weak $\phi-\eta$ coupling. Massiveness of the $\phi$ Higgs states would then imply massiveness of the corresponding $\eta$ Higgs states, with only one neutral and two charged pseudo Goldstone Higgs states potentially observable at LHC energies. In such models, one simultaneously has observable light Higgs states (the pseudo Goldstone triplet) and "new physics" implied by the strongly self-coupled $\phi$ and $\eta$ Higgs sectors.

\section{Acknowledgments}

This work was supported in part by the Department of Energy under Grant \#DEFG02-90ER40542. I wish to thank the members of the SLAC Theory Group for stimulating comments on a seminar I gave on Ref. [1], which led to the writing of this paper. I also wish

to thank C. Bernard, B. Mc Kellar, P. Mc Kenzie, C. Quigg, S. Sharpe, and S. B. Treiman for helpful conversations or email correspondence. 


\section{References}

[1] S. L. Adler, Phys. Rev. D 59, 015012 (1999), hep-ph/9806518.

[2] M. Peskin, private communication.

[3] S. L. Adler, Erratum for Ref. [1], Phys. Rev. D (in press), and available by email request to "adler @ias.edu".

[4] S. L. Glashow and S. Weinberg, Phys. Rev D 15, 1958 (1977); B. Mc Williams and L.-F. Li, Nucl. Phys. B179, 62 (1981); O. Shankar, Nucl. Phys. B206, 253 (1982).

[5] M. K. Gaillard and B. W. Lee, Phys. Rev. D 10, 897 (1974), equation proceeding Eq. (2.8).

[6] B. Mc Williams and O. Shanker, Phys. Rev. D 22, 2853 (1980).

[7] C. R. Allton, et. al., " $B$-parameters for $\Delta S=2$ Supersymmetric Operators", heplat/9806016.

[8] Particle Data Group, Eur. Phys. J. C 3, 1 (1998).

[9] A. X. El-Khadra, A. S. Kronfeld, P. B. Mackenzie, S. M. Ryan, and J. N. Simone, Phys. Rev. D 58, 014506 (1998).

[10] S. Dawson, "Introduction to Electroweak Symmetry Breaking", hep-ph/9901280.

[11] R. Gatto, G. Morchio, and F. Strocchi, Phys. Letters 83B, 348 (1979); G. Segrè and H. A. Weldon, Ann. Phys. (NY) 124, 37 (1980). 
Table I. Six-Higgs doublet model fit to experimental data: second case of Sec. V

$\begin{array}{ccc}\text { quantity } & \text { target value } & \text { fitted value } \\ & & \\ M_{u} & 0.0033 & 0.0033 \\ M_{c} & 1.25 & 1.26 \\ M_{t} & 173.8 & 174.0 \\ & & \\ M_{d} & 0.0060 & 0.0064 \\ M_{s} & 0.115 & 0.111 \\ M_{b} & 4.25 & 4.24 \\ & & \\ M_{e} & 0.00051 & 0.00051 \\ M_{\mu} & 0.1057 & 0.1057 \\ M_{\tau} & 1.777 & 1.777 \\ & & \\ & & \\ s_{12} & 0.221 & 0.221 \\ s_{13} & 0.0059 & 0.0062 \\ s_{23} & 0.039 & 0.037 \\ \sin \delta_{13} \mid & 0.60 & 0.48\end{array}$


Table II. Six-Higgs doublet model fit to experimental data:

third case of Sec. V

$\begin{array}{ccc}\text { quantity } & \text { target value } & \text { fitted value } \\ M_{u} & 0.0033 & 0.0032 \\ M_{c} & 1.25 & 1.25 \\ M_{t} & 173.8 & 173.9 \\ & & \\ M_{d} & 0.0060 & 0.0065 \\ M_{s} & 0.115 & 0.096 \\ M_{b} & 4.25 & 4.25 \\ & & \\ M_{e} & 0.00051 & 0.00051 \\ M_{\mu} & 0.1057 & 0.1057 \\ M_{\tau} & 1.777 & 1.777 \\ & & \\ & & \\ s_{12} & 0.221 & 0.221 \\ s_{13} & 0.0059 & 0.0059 \\ s_{23} & 0.039 & 0.039 \\ \left|\sin \delta_{13}\right| & 0.60 & 0.33\end{array}$

